\title{
Weak-stripe magnetic domain evolution with an in-plane field in epitaxial FePd thin films: Model versus experimental results
}

\author{
A. Marty, ${ }^{\text {a) }}$ Y. Samson, B. Gilles, and M. Belakhovsky \\ CEA Grenoble, Département de Recherche Fondamentale sur la Matière Condensée, SP2M, \\ ave des Martyrs, 38054 Grenoble cedex 9, France
}

E. Dudzik, H. Dürr, S. S. Dhesi, and G. van der Laan

Daresbury Laboratory, Warrington WA4 4AD, United Kingdom

\section{J. B. Goedkoop}

University of Amsterdam, NL-1018 XE Amsterdam

The magnetic domain structure in thin ferromagnetic films with perpendicular anisotropy is investigated. The effect of an in-plane magnetic field on the presence of domains and the magnetization profile is analyzed within the framework of a one-dimensional analytical model. The general results are reported in a state diagram as a function of the two relevant dimensionless parameters: the quality factor and the ratio of the film thickness to the exchange length. The experimental results, including hysteresis measurements, magnetic force microscopy, and x-ray resonant magnetic scattering obtained on a generic FePd thin layer, are reported and compared to the model. () 2000 American Institute of Physics. [S0021-8979(00)65508-1]

\section{INTRODUCTION}

In magnetic thin films with a perpendicular anisotropy of medium strength (quality factor $Q$ close to 1 ) the magnetic structure exhibits a transition between in-plane and out-ofplane magnetization. The latter consists of a periodic modulation of the perpendicular component of the magnetization leading to parallel stripe domains. The transition and the period of the stripe structure both depend on the relevant magnetic parameters of the material (anisotropy constant, saturation magnetization, and exchange constant), the thickness of the magnetic layer, and the applied field. A considerable number of theoretical works have been devoted to the analysis of the stability of various magnetic structures in such films. By solving numerically the complete set of micromagnetic equations (Ref. 1 and references therein), a precise insight into the magnetic configuration can be obtained. However, analytical models based on the variation of a onedimensional (1D) magnetization allow us to get a fast, although crude, overview of the general behavior of magnetic stripes allowing the construction of state diagrams. In these models, the magnetization is assumed to remain in the vertical plane parallel to the stripes and to vary only with the in-plane direction $x$ perpendicular to the stripes. The equilibrium state is found by minimizing the magnetic energy with respect to $\theta(x)$, where $\theta$ is the angle between the magnetization vector and the film plane. This approach was used by Kaczer $^{2}$ who introduced the Jacobi sine function to parameterize the profile function, $\sin [\theta(x)]=s \operatorname{Sn}(x / \delta, s)$, where $\delta$ is the fixed standard Bloch wall thickness and $s$ is the shape variable to be optimized. Later, Sukstanskii ${ }^{3}$ also proposed to leave $\delta$ free. However, these authors did not take into account any applied magnetic field. Druyvesteyn ${ }^{4}$ proposed a

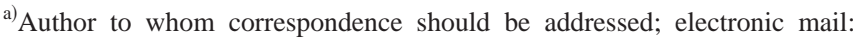
amarty@cea.fr
1D model involving an in-plane field, but the dipolar energy was only valid for square profiles. In the present work, we intend to generalize the Sukstanskii approach by including the effect of an in-plane field on the stripes' stability and on their magnetization profile.

\section{MODEL IN THE PRESENCE OF AN IN-PLANE MAGNETIC FIELD}

The trial function used by Sukstanskii ${ }^{3}$ linked the shape parameter $s$ to the maximum angle, $\theta_{m} ; s=\sin \left(\theta_{m}\right)$. Here the trial function has to be more general in order to make the maximum angle independent of $s$. This is needed when an in-plane field leads to values of $\theta_{m}$ far below $90^{\circ}$ while the magnetization profile can remain close to a square one in the case of a large perpendicular anisotropy. Then the $s$ factor of the Jacobi sine function is replaced by an independent parameter $m=\sin \left(\theta_{m}\right)$

$$
\frac{M_{z}(x)}{M_{s}} \equiv m_{z}(x)=\sin (\theta(x))=m \times \operatorname{Sn}(4 K(s) x / p, s) .
$$

The elliptic function $K(s)$ allows us to normalize the first argument of $\mathrm{Sn}$ to the period $p$. Figure 1 shows the evolution of the shape of the function $\operatorname{Sn}(4 K(s) x, s)$ for several values

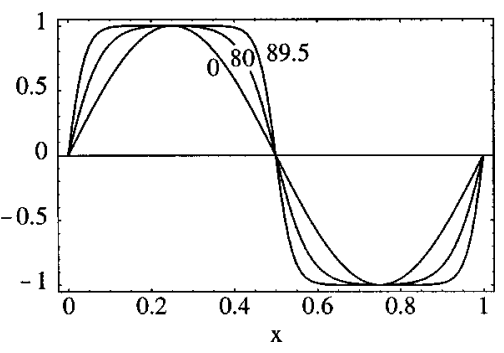

FIG. 1. Profile of the Jacobi sine function, $\mathrm{Sn}$, for various parameters $\theta_{s}$ (deg) where $s=\sin \left(\theta_{s}\right)$. 
of $\theta_{s}$, where $s=\sin \left(\theta_{s}\right)$. The total magnetic energy is the sum of the exchange, anisotropy, Zeeman, and dipolar energies. In the 1D scheme, it can be written using dimensionless parameters

$$
\begin{aligned}
e_{\mathrm{tot}}= & \frac{1}{t^{2}} \int_{0}^{p}\left[h \partial_{x} \theta(x)\right]^{2} \frac{d x}{p}+Q \int_{0}^{p} \cos ^{2}[\theta(x)] \frac{d x}{p} \\
& -2 h_{a} \int_{0}^{p} \cos [\theta(x)] \frac{d x}{p}+e_{\mathrm{dip}},
\end{aligned}
$$

where the parameters $t=h /\left(A / 2 \pi M_{s}^{2}\right)^{1 / 2}$ (dimensionless thickness), $Q=K_{u} / 2 \pi M_{s}^{2}$ (quality factor), $h_{a}=H_{a} / 4 \pi M_{s}$ (dimensionless field), and $u=p / 2 h$ (dimensionless period) are related to the physical parameters: $M_{s}$ (saturation magnetization), $K_{u}$ (uniaxial anisotropy constant), $A$ (exchange constant), and $h$ (layer thickness). In the following, the dimensionless parameters: $t, Q, u$, and $h_{a}$ will be used instead of their dimensioned counterparts: $h, K_{u}, p$, and $H_{a}$. With the trial function (1), the three definite integrals in Eq. (2) may be expressed analytically in terms of the complete elliptic integrals $K, E$, and $\Pi$, as given in the Appendix together with the dipolar energy. The dipolar energy is an infinite series corresponding to the sum of the contributions of the odd Fourier components of the Jacobi sine function. It corresponds to the expression used by Sukstanskii, multiplied by a $(\mathrm{m} / \mathrm{s})^{2}$ factor.

\section{CRITICAL LINE IN THE STABILITY DIAGRAM FOR THE APPEARANCE OF A STRIPE PATTERN}

The stability of stripes versus in-plane magnetization must be investigated first. This was done by Sukstankii ${ }^{3}$ for the zero field case. He provided a parametric expression relating the critical thickness to the anisotropy above which the in-plane configuration was unstable "with respect to", (w.r.t.) stripes. When a finite in-plane field is present, this transition occurs, for a given set $(Q, t)$, at a saturation field, $h_{\text {sat }}$. Just below $h_{\text {sat }}$ the in-plane configuration is unstable with respect to infinitesimal undulations of $m_{z}(x)$, of period $u$. This may be expressed mathematically as

$$
\begin{aligned}
& \left(\partial^{2} e_{\text {tot }} / \partial m^{2}\right)_{m=0}=0 \\
& \left(\partial^{3} e_{\text {tot }} / \partial m^{2} \partial u\right)_{m=0}=0 .
\end{aligned}
$$

To investigate this second-order-like transition, only a sinusoidal trial function is needed $\left[m_{z}=m \sin (2 \pi x / p)\right]$. Solving these equations leads to the parametric expressions relating the quality factor, the saturation field, and the thickness of the layer

$$
\begin{aligned}
& Q-h_{\mathrm{sat}}=\frac{3 u-(\pi+3 u) e^{-\pi / u}}{2 \pi}, \\
& t=\sqrt{2 \pi^{3}} / u \sqrt{u-(\pi+u) e^{-\pi / u}} .
\end{aligned}
$$

It is remarkable that our generalization leads to equations identical to the ones in Ref. 3, provided that $Q$ is replaced by $Q-h_{\text {sat }}$. Graphically, Eq. (4) may be represented as a simple translation of the critical line shown in Fig. 2.

On the right of the critical line, stripes develop. Their period and the maximum angle, $\theta_{m}$ of the magnetization may

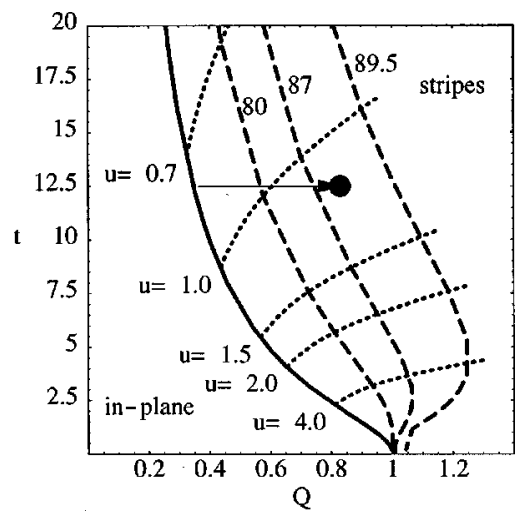

FIG. 2. States diagram in the $(Q, t)$ plane: critical line (solid line), constant period lines (dotted lines), constant max angle $\theta_{m}$ in degrees (dashed lines), our sample (disk), saturation field for our sample (horizontal arrow).

be computed from the minimization of $e_{\text {tot }}$ with respect to the period, $u$, and the profile parameters, $m$ and $s$. Constant period lines $(u=c t e)$ and constant maximum angle lines $(m$ $=\sin \left(\theta_{m}\right)=c t e$ ) have been computed for zero field. They are shown in Fig. 2. It can be seen that the maximum angle, $\theta_{m}$, increases steeply near the critical line and then more slowly further from this line. For very thin layers, $t \ll 1$, stripes appear only for $Q>1$, the maximum angle rises sharply to $90^{\circ}$ and the profile is close to a square pattern. For this latter situation, the simpler model of Kaplan and Gehring ${ }^{5}$ dedicated to ultrathin films is well suited.

\section{APPLICATION TO EXPERIMENTAL RESULTS OBTAINED ON STRIPPED FePd FILMS}

In the light of the above model, we can now discuss experimental results obtained on FePd thin films. An equiatomic FePd alloy layer of $40 \mathrm{~nm}$ thickness was epitaxially grown on a $\mathrm{MgO}(001)$ substrate at $220{ }^{\circ} \mathrm{C}$ within a molecular beam facility. To prevent oxidation, a $2 \mathrm{~nm} \mathrm{Pd}$ layer was deposited on top of the alloy layer. The growth process resulted in a (chemically) partially ordered FePd alloy leading to an intermediate anisotropy ( $Q$ close to 1$)$. More details on the growth process and extensive magnetic characterizations can be found in Ref. 6 and references therein. The in-plane hysteresis has been measured using a vibrating sample magnetometer (Fig. 3). The period has been estimated from magnetic force microscopy images performed in zero field and as a function of the in-plane field using soft $\mathrm{x}$-ray resonant

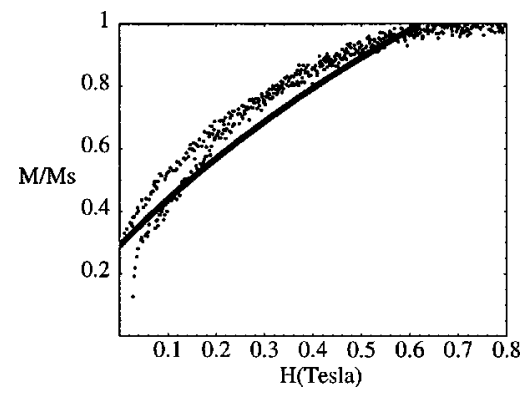

FIG. 3. In-plane magnetization curve, experimental (dots), and calculated for $Q=0.83$ and $t=12.5$ (solid line). 


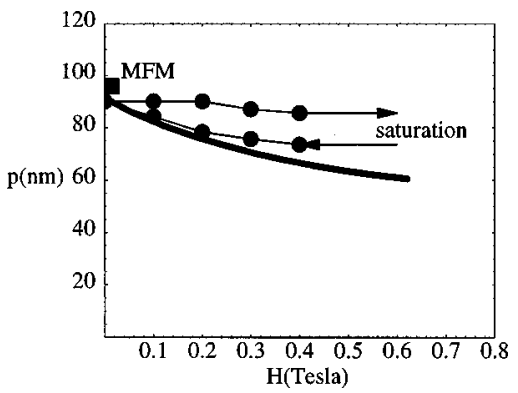

FIG. 4. Stripes period vs in-plane field, from x-ray resonant magnetic scattering experiment (dots), from magnetic force microscopy (square), calculation with $Q=0.83, t=12.5$, and $M_{s}=1030$ Oe (solid line).

magnetic scattering. ${ }^{7}$ These latter measurements were performed at the European Synchrotron Radiation Facility synchrotron source on the ID 12B beamline at grazing incidence $\left(5^{\circ}\right)$. The periodic stripes gave rise to satellite peaks in the vicinity of the specular peak. Their periods were deduced from the in-plane component of the scattering vector of the magnetic satellites, and are reported in Fig. 4 for in-plane field increasing to saturation and then decreasing to zero field.

Both the saturation magnetization and the exchange constant were taken from the bulk equiatomic FePd alloy Ref. 6 and reference therein: $M_{s}=1030 \mathrm{emu} / \mathrm{cm}^{3}$ and $A=6.9$ $\times 10^{-7} \mathrm{erg} / \mathrm{cm}$. This assumes that these two parameters do not depend strongly on the chemical order for a constant composition. The quality factor was deduced from the inplane saturation field (Fig. 3) and Eqs. (4) giving a value $Q=0.83$. The saturation field corresponds to the length of the arrow shown in Fig. 2, which joins the critical line to the black dot representing the sample state. The in-plane magnetization component and the period have been computed by minimizing $e_{\text {tot }}$ [Eq. (2)] as a function of the in-plane field. They are respectively shown in Figs. 3 and 4.
The agreement with the experimental results is rather good. Therefore this 1D model proves to be efficient in investigating the general behavior of stripes (period and canting angle). Its main limitation lies in the assumption of an in-depth magnetic homogeneity, since $\mathrm{SXMRS}^{7}$ has also shown that closure domains are present in this sample.

\section{APPENDIX}

Here follow the analytic expressions for the integrals in Eq. (2) and the dipolar energy for the trial function (1)

$$
\begin{aligned}
& \int_{0}^{p}\left[h \partial_{x} \theta(x)\right]^{2} \frac{d x}{p}=\frac{4 E(s)}{u^{2} m^{2}}\left[m^{2} E(s)-\left(1-m^{2}\right) s^{2} K(s)\right. \\
& \left.+\left(1-m^{2}\right)\left(s^{2}-m^{2}\right) \Pi(m, s)\right], \\
& \int_{0}^{p} \cos ^{2}[e(x)] \frac{d x}{p}=1-\frac{m^{2}}{s^{2}}+\frac{m^{2} E(s)}{s^{2} K(s)}, \\
& \int_{0}^{p} \cos [\theta(x)] \frac{d x}{p}=\frac{\pi\left(m / \sqrt{m^{2}-1}, \sqrt{s^{2}-m^{2}} / \sqrt{1-m^{2}}\right)}{K(s) \sqrt{1-m^{2}}}, \\
& e_{\mathrm{dip}}=\frac{m^{2}}{s^{2}} \frac{2 \pi u}{K^{2}(s)} \sum_{n=1,3, \ldots}^{\infty} \frac{q^{n}[1-\exp (-\pi n / u)]}{n\left(1-q^{n}\right)^{2}} \\
& \text { with } q=\exp \left(-\pi K\left(\sqrt{1-s^{2}}\right) / K(s)\right) \text {. }
\end{aligned}
$$

${ }^{1}$ M. Labrune and J. Miltat, J. Appl. Phys. 75, 2156 (1994).

${ }^{2}$ J. Kaczer, M. Zeleny, and P. Suda, Czech. J. Phys., Sect. B 13, 579 (1963).

${ }^{3}$ A. L. Sukstanskii and K. I. Primak, J. Magn. Magn. Mater. 169, 31 (1997).

${ }^{4}$ W. F. Druyvesteyn, J. W. F. Dorleijn, and P. J. Rijnierse, J. Appl. Phys. 44, 2397 (1973).

${ }^{5}$ R. Kaplan and G. A. Gehring, J. Magn. Magn. Mater. 128, 11 (1993).

${ }^{6}$ V. Gehanno, R. Hoffmann, Y. Samson, A. Marty, and S. Auffret, Eur. Phys. J. B 10, 457 (1999).

${ }^{7}$ H. A. Dürr, E. Dudzik, S. S. Dhesi, J. B. Goedkoop, G. van der Laan, M. Belakhovsky, C. Mocuta, A. Marty, and Y. Samson, Science 284, 2166 (1999). 